\title{
Erholung verliert an Fahrt - Wirtschaft und Politik weiter im Zeichen der Pandemie
}

Infolge der Maßnahmen zur Eindämmung der Corona-Pandemie ist die deutsche Wirtschaftsleistung in der ersten Jahreshälfte drastisch gesunken, vor allem in den Monaten März und April. Schon im Mai setzte eine kräftige Gegenbewegung ein, die sich in nahezu allen Branchen bis zum aktuellen Rand fortsetzte. Dieser Erholungsprozess dürfte an Fahrt verlieren. Die Institute erwarten daher nach einem Rückgang des Bruttoinlandsprodukts um 5,4\% (2020) einen Zuwachs um 4,7\% (2021) und um 2,7\% im Jahr 2022. Sie revidieren damit ihre Prognose aufgrund des nunmehr etwas schwächeren Erholungsprozesses gegenüber dem Frühjahr nach unten. Die Wirtschaftspolitik hat frühzeitig mit massiven finanzpolitischen Maßnahmen auf die Corona-Krise reagiert. Die Konjunkturprogramme haben im Zusammenspiel mit den automatischen Stabilisatoren dazu beigetragen, dass die verfügbaren Einkommen der privaten Haushalte selbst in der akuten Krisenphase insgesamt stabil blieben.

Die Corona-Pandemie hat weite Teile der Weltwirtschaft im Frühjahr drastisch einbrechen lassen, wobei die Rückgänge vielerorts schärfer ausfielen als während der Großen Rezession 2008/2009. Der wirtschaftliche Einbruch verlief international weitgehend synchron. Sowohl in Europa und in den USA als auch in den großen Schwellenländern begann die gesamtwirtschaftliche Produktion mit der zunehmenden Zahl von Corona-Infektionen und als Folge zum Teil drastischer Eindämmungsmaßnahmen im März zu sinken. Der Abschwung verstärkte sich im April 2020, sodass sich der Rückgang des Bruttoinlandsprodukts (BIP) trotz der in den folgenden Monaten einsetzenden Erholung auf das zweite Quartal konzentrierte. Eine Ausnahme bildet China, dort sind der Epidemie- und der Konjunkturverlauf der übrigen Welt etwa ein Quartal voraus. Auch wenn die Pandemie noch keineswegs auf dem Rückzug ist, wurde weltweit bereits im Sommer ein Großteil der wirtschaftlichen Aktivität wieder aufgenommen. Mittlerweile dürfte ein Gutteil des Einbruchs wettgemacht worden sein.

Die der Prognose zugrunde liegende Annahme über den Pandemieverlauf sieht vor, dass in weiten Teilen der Welt die bestehenden Einschränkungen zunächst aufrechterhalten werden, wobei es örtlich und zeitlich begrenzt auch zu Verschärfungen kommen wird. Erst im Verlauf des kommenden Jahres gelingt es dann, die Pandemie zurückzu-

(C) Der/die Autor(en) 2020. Open Access: Dieser Artikel wird unter der Creative Commons Namensnennung 4.0 International Lizenz (https:// creativecommons.org/licenses/by/4.0/deed.de) veröffentlicht.

Open Access wird durch die ZBW - Leibniz-Informationszentrum Wirtschaft gefördert. drängen, sodass Eindämmungsmaßnahmen schrittweise aufgehoben werden können. Im Zuge dessen wird die wirtschaftliche Tätigkeit immer weniger behindert.

Zurzeit ist die Unsicherheit über den Pandemieverlauf und damit mögliche erneute Infektionsschutzmaßnahmen noch

Prof. Dr. Oliver Holtemöller ist stellvertretender Präsident und leitet die Abteilung Makroökonomik am Leibniz-Institut für Wirtschaftsforschung Halle.

Prof. Dr. Stefan Kooths leitet das Prognosezentrum am Institut für Weltwirtschaft in Kiel.

Dr. Claus Michelsen leitet die Abteilung Konjunkturpolitik am Deutschen Institut für Wirtschaftsforschung in Berlin (DIW Berlin).

Prof. Dr. Torsten Schmidt ist Leiter des Kompetenzbereichs „Wachstum, Konjunktur, Öffentliche Finanzen“ am RWI - Leibniz-Institut für Wirtschaftsforschung in Essen.

Prof. Dr. Timo Wollmershäuser ist stellvertretender Leiter des ifo Zentrums für Makroökonomik und Befragungen in München. 
groß. Diese Unsicherheit beeinträchtigt insbesondere die Investitionen von Unternehmen und die Bereitschaft von privaten Haushalten, größere Anschaffungen zu tätigen. Der private Konsum wird in vielen Ländern zwar dadurch gestützt, dass sich infolge verringerter Konsummöglichkeiten in erheblichem Umfang Kaufkraft aufgestaut hat, doch wird er vielerorts gleichzeitig durch eine stark gestiegene Arbeitslosigkeit gebremst. Die Normalisierung der wirtschaftlichen Tätigkeit, die sich aus den Lockerungen der Eindämmungsmaßnahmen ergibt, wird aber wohl auf absehbare Zeit nicht zu einer vollständigen Rückkehr auf den vor der Krise erwarteten Wachstumstrend führen. So dürfte die Investitionsneigung unter anderem infolge von verschlechterten Absatzerwartungen und einer reduzierten Eigenkapitalbasis nachhaltig gedämpft bleiben. Dies führt zu einem geringeren Wachstum des Kapitalstocks, der zudem im Zuge des zu erwartenden deutlichen Anstiegs von Insolvenzen in Teilen vermutlich abgeschrieben werden muss. In der Krise abgebaute Arbeitsplätze werden nur allmählich an anderer Stelle neu entstehen und die Arbeitskräfte nicht in allen Fällen wieder mit der zuvor verzeichneten Produktivität beschäftigt werden können, da die Anpassung der Qualifikation Zeit erfordert.

\section{Weltwirtschaft}

All dies belastet zunächst die weitere globale Erholung, die sich nach dem Zwischenspurt um die Jahresmitte wohl deutlich verlangsamen wird. Die Weltproduktion dürfte im Jahresdurchschnitt 2020 um 4,0\% sinken und damit so stark wie noch nie seit dem Zweiten Weltkrieg (vgl. Abbildung 1). Für 2021 erwarten die Institute aufgrund des fortschreitenden Erholungsprozesses eine kräftige Zunahme um 5,9\%. Im Jahr 2022 dürfte die Weltproduktion mit 3,7\% expandieren, womit sich die Veränderungsraten allmählich normalisieren. Aus Sicht der deutschen Exportindustrie stellt sich die Entwicklung noch ungünstiger dar: der weltwirtschaftliche Einbruch im laufenden Jahr ist mit 5,6\% merklich stärker und die Erholung mit Raten von 5,7\% (2021) und 3,4\% (2022) etwas verhaltener. Insgesamt bleibt damit die wirtschaftliche Aktivität im gesamten Prognosezeitraum und darüber hinaus deutlich unter den Werten, die ohne Pandemie zu erwarten gewesen wären.

Im Welthandel waren im Juli 2020 bereits rund zwei Drittel des zwischen Ende 2019 und Mai 2020 verzeichneten Rückgangs um $17 \%$ wieder aufgeholt. Der Beginn der Erholung im Mai fiel mit einem Rückgang der Neuinfektionen in vielen Ländern und der damit verbundenen schrittweisen Lockerung der Infektionsschutzmaßnahmen zusammen. Zuletzt scheint die Erholung wohl auch deshalb an Schwung verloren zu haben, weil die Infektionszahlen vielerorts wieder deutlich gestiegen sind. In den kommenden Monaten dürfte sich die Erholung mit schwächerer Dyna-

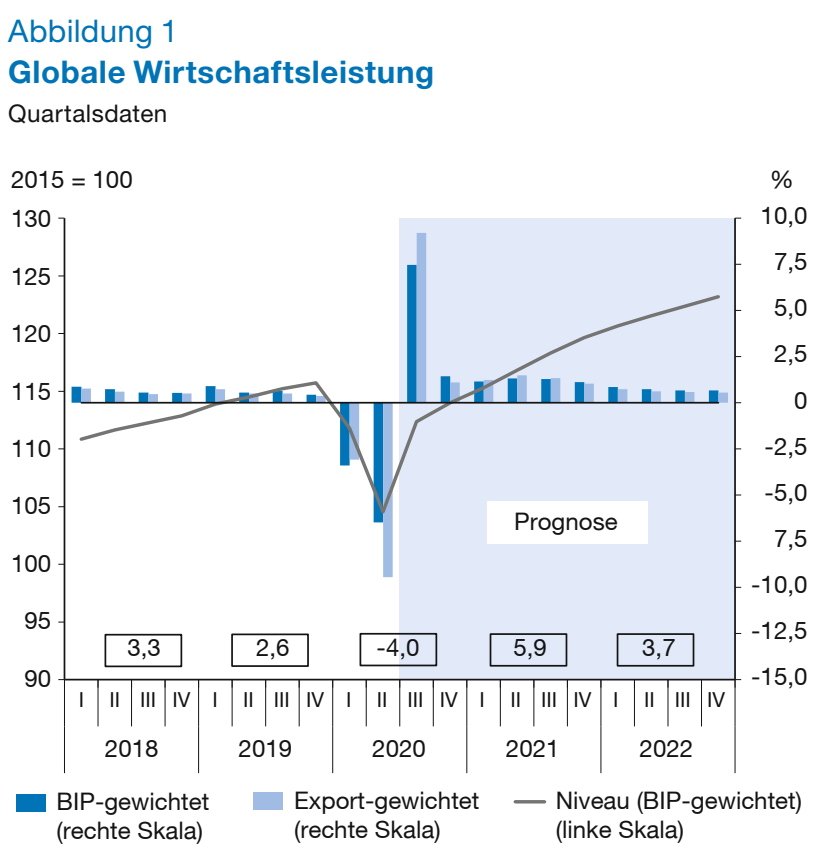

Anmerkung: Aggregat aus 51 Ländern. Gewichtet mit dem Bruttoinlandsprodukt von 2018 in US-Dollar bzw. dem Anteil an deutschen Exporten. Linie: Niveau (Index, linke Skala); Balken: Veränderung gegenüber Vorquartal (rechte Skala); gerahmt: Veränderung gegenüber Vorjahr in \% (BIPgewichtet).

Quellen: IWF; OECD; nationale Statistikämter; Berechnungen der Institute; ab 3. Quartal 2020: Prognose der Institute.

mik fortsetzen. Gleichwohl wird der Welthandel im Gesamtjahr 2020 um 6,9\% unter seinem Vorjahresniveau bleiben. In den Jahren 2021 und 2022 dürfte er um 5,7\% bzw. 3,1\% ausgeweitet werden.

Die Weltwirtschaft ist im Sommer vor allem deshalb angesprungen, weil viele wirtschaftliche Aktivitäten, die mit sozialen Kontakten einhergehen, wieder möglich wurden und die Kaufkraft der privaten Haushalte weitaus weniger zurückging als die Produktion. Hier dürften eine aktive Finanzpolitik sowie automatische Stabilisatoren vor allem in den fortgeschrittenen Volkswirtschaften eine entscheidende Rolle gespielt haben. Beschäftigte, die aufgrund der Pandemie ihre Lohneinkommen verloren haben, werden in den USA und in vielen Ländern Europas in erheblichem Ausmaß finanziell unterstützt. Unternehmen, die aufgrund von Infektionsschutzmaßnahmen Umsatzausfälle verkraften müssen, wird Liquidität bereitgestellt, oder die Staaten bürgen für Unternehmenskredite. Zudem sind erhöhte öffentliche Investitionen und Maßnahmen zur Förderung privater Investitionen geplant. Die in den fortgeschrittenen Volkswirtschaften geschnürten Fiskalpakete sind in ihrem Umfang einmalig. In den USA ist für 2020 mit einem öffentlichen Budgetdefizit von mehr als $15 \%$ im Verhältnis zum BIP zu rechnen, und im Euroraum dürfte die Defizitquote reichlich $8 \%$ betragen. Expansive finanzpolitische Maßnahmen von 
erheblichem Umfang wurden auch in großen Schwellenländern wie China, Brasilien und Indien ergriffen.

Die Zentralbanken in den großen fortgeschrittenen Volkswirtschaften unterstützen die finanzpolitischen Maßnahmen, indem sie in großem Umfang Staatstitel erwerben und signalisieren, dass für längere Zeit mit Leitzinsen von um die $0 \%$ zu rechnen ist. Hiermit stabilisieren sie zugleich die Finanzmärkte. Wo die Leitzinsen zu Beginn des Jahres 2020 nennenswert über $0 \%$ lagen, sind sie in Reaktion auf die Pandemie gesenkt worden, und zwar auch in den meisten Schwellenländern recht deutlich. Ende August 2020 hat die US-Notenbank darüber hinaus Änderungen an ihrer geldpolitischen Strategie bekanntgegeben. Neu ist vor allem, dass die Notenbank plant, im Falle einer längeren Phase mit Inflationsraten unter dem $2 \%$-Ziel (wie gegenwärtig) für einige Zeit auch Inflationsraten anzustreben, die moderat über dem Ziel liegen.

\section{Wirtschaftliche Entwicklung in Deutschland}

Die Wirtschaftsleistung in Deutschland ist infolge der Maßnahmen zur Eindämmung der Corona-Pandemie in der ersten Jahreshälfte 2020 so stark eingebrochen wie nie zuvor seit Bestehen der Bundesrepublik, wobei sich der Einbruch auf die Monate März und April konzentrierte (vgl. Abbildung2). Im zweiten Quartal wurde das Vorkrisenniveau des BIP um 11,5\% unterschritten. Im Mai setzte aber bereits eine kräftige Gegenbewegung ein, die sich in nahezu allen Branchen bis zum aktuellen Rand fortsetzte. Für das dritte Quartal rechnen die Institute mit einem deutlichen Zuwachs des BIP um 6,5\%. Die Wirtschaftsleistung liegt damit aber immer noch fast $6 \%$ unter dem Niveau vom Schlussquartal des Jahres 2019. Dieser Erholungsprozess dürfte zunehmend an Fahrt verlieren. Denn Nachholeffekte laufen aus, einige Branchen sind weiterhin erheblichen Einschränkungen ausgesetzt, und die für die deutsche Wirtschaft wichtige globale Investitionstätigkeit dürfte noch für einige Zeit geschwächt bleiben.

Die Institute revidieren ihre Prognose für das BIP gegenüber dem Frühjahrsgutachten für das laufende und das kommende Jahr um jeweils gut 1 Prozentpunkt nach unten. Grund dafür ist, dass der weitere Erholungsprozess nunmehr etwas schwächer eingeschätzt wird als noch im Frühjahr. Die Wirtschaftsleistung dürfte 2020 um 5,4\% schrumpfen und 2021 um 4,7 \% zulegen. Für das Jahr 2022 erwarten die Institute einen Zuwachs um 2,7\%. Das Vorkrisenniveau wird voraussichtlich erstmals im Schlussquartal des kommenden Jahres wieder überschritten. Damit sind die Folgen der Corona-Krise aber bei weitem noch nicht ausgestanden. So liegt die Wirtschaftsleistung dann immer noch 2,5\% unter dem Niveau, das die Institute im Herbst 2019, als die Pandemie noch nicht absehbar war, erwartet
Abbildung 2

Bruttoinlandsprodukt (Deutschland)
Quartalsdaten

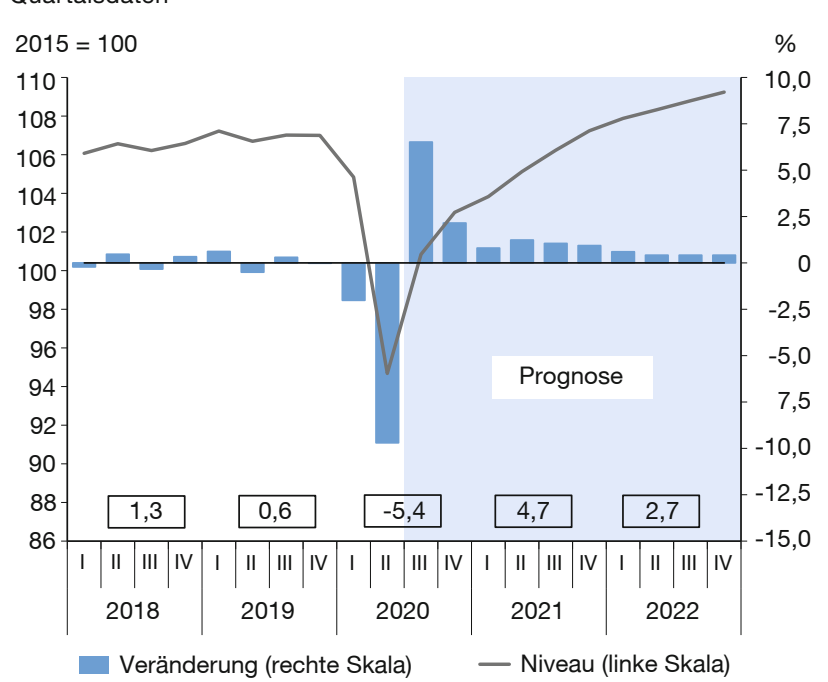

Anmerkung: Quartalsdaten: preis-, kalender- und saisonbereinigt; Linie: Niveau (Index, linke Skala); Balken: Veränderung gegenüber Vorquartal (rechte Skala); gerahmt: jahresdurchschnittliche preisbereinigte Veränderung in \%.

Quellen: Statistisches Bundesamt; Prognose der Institute.

hatten. Die Normalauslastung der gesamtwirtschaftlichen Produktionskapazitäten dürfte erst Ende des Jahres 2022 in etwa erreicht werden - und das auch nur bei einem dann gegenüber dem vergangenen Herbstgutachten um 1,3\% niedriger veranschlagten Niveau des Produktionspotenzials. Diese Einschätzung ist allerdings mit besonderer Unsicherheit behaftet, weil sich derzeit kaum absehen lässt, welche langfristigen Schäden die Corona-Krise in den Wirtschaftsstrukturen hinterlassen wird und wie die wirtschaftspolitischen Reaktionen wirken.

Im Vergleich zur Großen Rezession bestehen trotz ähnlicher Jahresraten für das BIP in den Jahren 2009 und 2020 wichtige Unterschiede. So ging dem Einbruch in der zweiten Jahreshälfte 2008 ein ausgeprägter Boom voraus, während die deutsche Wirtschaft am Ende des Jahres 2019 schon zwei Jahre im Abschwung war. Allerdings bestehen gute Chancen, dass die Erholung diesmal zügiger verläuft. Denn der Weltfinanzkrise gingen langjährige Fehlentwicklungen im Wirtschaftssystem voraus, deren Korrektur längere Zeit erforderte. Dagegen hat die Corona-Pandemie die Weltwirtschaft als exogener Schock getroffen, der die wirtschaftliche Aktivität zwar zwischenzeitlich massiv unterbrach, die Produktionsstrukturen aber in deutlich geringerem Umfang infrage stellen dürfte. Dies erklärt, weshalb die Wirtschaftsleistung nach der Shutdown-Phase dort, wo es das Pandemiegeschehen zulässt, rascher wieder aus dem 
tiefen Tal herausfindet als im Nachgang der Weltfinanzkrise. Zeigte sich in der Großen Rezession ein u-förmiger Verlauf bei der deutschen Wirtschaftsleistung, die erst nach zwölf Quartalen das Vorkrisenniveau überstieg, zeichnet sich für die Corona-Krise eher ein v-förmiger Verlauf ab, bei dem ein Jahr früher zum Vorkrisenniveau aufgeschlossen werden kann. Gleichwohl stellt der verbleibende Aufholprozess die mühsamere Wegstrecke dar, sodass sich der rechte Schenkel der V-Formation deutlich abflacht.

Die Große Rezession war für die deutsche Konjunktur vor allem eine Export- und Investitionskrise, während in der Corona-Pandemie zusätzlich und in massiver Weise auch der private Konsum in Mitleidenschaft gezogen wurde. Maßgeblich hierfür sind fehlende Konsummöglichkeiten infolge behördlicher und privater Vorsichtsmaßnahmen zum Infektionsschutz. Weil die verfügbaren Einkommen von den automatischen Stabilisatoren und finanzpolitischen Maßnahmen gestützt wurden, schnellte die Sparquote der privaten Haushalte im zweiten Quartal auf ein historisches Niveau von $21 \%$ hoch. In diesem und im kommenden Jahr dürften insgesamt etwa 150 Mrd. Euro zusätzlich gespart werden. In dem Maße, in dem Infektionsschutzmaßnahmen gelockert werden, wird sich mit den Konsummöglichkeiten auch die Sparquote nach und nach normalisieren.

Die Corona-Krise hinterlässt deutliche Spuren am Arbeitsmarkt. So sind trotz der massiven Inanspruchnahme der Kurzarbeit bis zum August 670.000 Arbeitsplätze weggefallen; stellt man in Rechnung, dass es ohne den CoronaSchock Beschäftigungszuwächse gegeben hätte, so steigt die Zahl der krisenbedingten Arbeitsplatzverluste nach Einschätzung der Institute auf 820.000. Die Arbeitslosenquote ist merklich gestiegen. Im Sommer 2020 war sie mit 6,4\% so hoch wie zuletzt vor fünf Jahren und 1,4 Prozentpunkte höher als zu Jahresanfang. Die Talsohle am Arbeitsmarkt scheint seitdem aber durchschritten zu sein. So deuten die Arbeitsmarktdaten am aktuellen Rand wieder auf einen allmählichen Beschäftigungsanstieg hin. Die Zahl der Erwerbstätigen dürfte das Vorkrisenniveau gleichwohl erst zur Mitte des Jahres 2022 wieder erreichen. Das Arbeitsvolumen bleibt hingegen auch am Ende des Prognosezeitraums noch leicht dahinter zurück.

\section{Risiken}

Neben dem ungewissen Verlauf der Corona-Pandemie ergeben sich aus dem internationalen Umfeld Risiken für die Prognose. So bergen die bereits eingetretenen Pandemiefolgen die Gefahr, dass der kräftige Anstieg der Staatsverschuldung infolge wegbrechender Staatseinnahmen und der massiven fiskalischen Stimulierungsmaßnahmen die Risikoprämien für manche Staaten so weit steigen lässt, dass die Finanzpolitik schon bald von Expansion auf Kon- solidierung schwenken muss. Auch besteht das Risiko, dass weltweit viele Unternehmen nach dem Auslaufen entsprechender Moratorien Insolvenz anmelden und zusätzliche Arbeitsplätze verloren gehen. Dies könnte auch die kreditgebenden Banken in Schwierigkeiten bringen und eine Finanzkrise auslösen. Diese Gefahr dürfte vor allem in vielen Schwellenländern bestehen.

Ferner schwelt nach wie vor die Gefahr sich verschärfender Handelskonflikte, insbesondere zwischen den USA und China. Latent ist zudem der Handelsstreit zwischen den USA und der Europäischen Union. Schließlich ist offen, welches Handelsregime zwischen der EU und Großbritannien gelten wird, wenn Ende 2020 die Übergangsphase im Austrittsprozess ausläuft. Hierzu ist für diese Prognose unterstellt, dass sich Großbritannien und die Europäische Union nicht zeitgerecht auf ein Handelsabkommen werden einigen können, dass aber pragmatische Ad-hoc-Regeln auf beiden Seiten eine gravierende Störung der Wirtschaftsbeziehungen verhindern werden. Zusätzliche Risiken resultieren aus heimischen Unwägbarkeiten. So ist unsicher, wie viele Unternehmen durch die Pandemie in ihrer Existenz bedroht sind. Denn die bis Ende September ausgesetzte Insolvenzanzeigepflicht erschwert die Diagnose mit Blick auf die Stabilität des Unternehmenssektors. Damit werden sich die Auswirkungen der Corona-Krise auf die Solvenz der Unternehmen erst nach dem dritten Quartal 2020 deutlicher abzeichnen. Sollte es dann zu einer größeren Insolvenzwelle kommen, würde sich dies in einem Anstieg der Arbeitslosigkeit und einem Rückgang der Kurzarbeit bemerkbar machen. Damit würde die Zahl der Erwerbstätigen vorübergehend schwächer steigen oder sogar sinken. Ein Anschwellen der Insolvenzfälle droht vor allem in besonders schwer betroffenen Branchen wie dem Gastgewerbe, aber auch generell im Segment der kleineren Unternehmen, die ihre Eigenkapitalposition in den Jahren vor der Krise weniger verbessert hatten als mittlere und große Unternehmen. Zwar dürften die Unternehmensgewinne im Zuge der Corona-Krise weniger stark einbrechen als während der Großen Rezession - auch infolge stützender staatlicher Maßnahmen -, allerdings waren sie in Relation zur Wirtschaftsleistung bereits vor der Pandemie längere Zeit sehr niedrig. Ein bedeutendes Aufwärtsrisiko für die Prognose ergibt sich aus der in erheblichem Umfang zurückgestauten Kaufkraft bei den privaten Haushalten. Diese ist durch die über längere Zeit eingeschränkten Konsummöglichkeiten bedingt. Sollten die privaten Haushalte - anders als von den Instituten unterstellt - verstärkt auf diese Ersparnisse zurückgreifen, würden hiervon zusätzliche Konjunkturimpulse ausgehen und der private Verbrauch könnte rascher zu seinem Vorkrisenniveau aufschließen. Je höher die konsumnahen Wirtschaftsbereiche ausgelastet sind, auf die diese zusätzliche Nachfrage träfe, desto stärker würde sich dies in einem Anstieg der Verbraucherpreise bemerkbar machen. 


\section{Wirtschaftspolitik}

Die Finanzpolitik hat im laufenden Jahr mit einem Bündel konjunkturstabilisierender Maßnahmen von beispiellosem Umfang auf die Krise reagiert. Die Konjunkturprogramme haben im Zusammenspiel mit den automatischen Stabilisatoren dazu beigetragen, dass die verfügbaren Einkommen der privaten Haushalte selbst in der akuten Krisenphase insgesamt stabil blieben. Auch deshalb dürfte der Gesamtstaat das laufende Jahr 2020 mit einem Rekorddefizit von 183 Mrd. Euro abschließen (5,5\% in Relation zur Wirtschaftsleistung), nach einem Überschuss von 53 Mrd. Euro (1,5\%) 2019. Während sich das konjunkturell bedingte Defizit im Zuge der Erholung 2021 verringert, bleibt das strukturelle Defizit mit 2,3\% deutlich erhöht. Der Fehlbetrag der öffentlichen Hand sinkt im Jahr 2021 auf 118 Mrd. Euro, bleibt aber mit 3,3\% immer noch leicht über der Maastricht-Grenze. Diese dürfte erst im übernächsten Jahr wieder unterschritten werden, allerdings fallen das Staatsdefizit mit 92 Mrd. Euro (2,5\%) und das strukturelle Defizit mit 2,2\% dann immer noch beträchtlich aus.

Der gesamtwirtschaftliche Einkommensausfall der Jahre 2020 und 2021 lässt sich auch durch die besten wirtschaftspolitischen Maßnahmen nicht wettmachen. Die Wirtschaftspolitik hat allerdings großen Einfluss darauf, welche Bevölkerungsgruppen die Einkommenseinbußen tragen werden und inwiefern der langfristige Einkommenstrend durch die Folgen der Pandemie beeinflusst wird. Angesichts der angebots- und nachfrageseitigen wirtschaftlichen Beeinträchtigungen infolge der CoronaPandemie und durch die damit verbundenen Infektionsschutzmaßnahmen begrüßen die Institute, dass die Wirtschaftspolitik diskretionäre Maßnahmen ergriffen hat, die auf die Stabilisierung der Konjunktur und auf die Vermeidung von negativen Langfristfolgen für die Wirtschaft abzielen. Insbesondere diejenigen Maßnahmen, die Einkommensausfälle der privaten Haushalte ersetzen, wie das Kurzarbeitergeld, und welche die von der Corona-Krise betroffenen Unternehmen direkt stützen, werden diesem Ziel gerecht.

Auch in Zeiten negativer Zinsen und großer Nachfrage nach Staatsschuldpapieren, die einen großen finanziellen Spielraum für die öffentliche Hand bieten, sollte der Einsatz öffentlicher Mittel auf Effizienz in dem Sinne bedacht sein, dass jeweils das Instrument zur Erreichung eines spezifischen Ziels eingesetzt wird, mit dem die geringsten Verzerrungen und Nebenwirkungen einhergehen. Diejenigen Maßnahmen des Konjunkturprogramms, die auf die Förderung des Konsums aller privaten Haushalte abzielen, erfüllen dieses Kriterium nicht. Besser wäre es, die Stimulierung der gesamtwirtschaftlichen Nachfrage über die automatischen Stabilisatoren hinaus vor allem mit gerichteten Transfers an diejenigen privaten Haushalte und Unternehmen vorzunehmen, die tatsächlich Einkommenseinbußen durch die Krise erlitten haben. Zum einen stabilisieren solche Transfers die Konjunktur mehr als unspezifische einnahmeseitige Maßnahmen wie die temporäre Mehrwertsteuersenkung und zum anderen verstärken sie nicht den Zielkonflikt zwischen Nachfragestabilisierung und Verteilung der Krisenkosten nach individueller Leistungsfähigkeit. Insbesondere mit Blick auf die Lastverteilung sehen die Institute daher Spielräume für Verbesserungen, die zugleich die Stabilisierungswirkung verstärken würden.

Das DIW Berlin hingegen beurteilt die im Frühjahr ergriffenen Soforthilfen und das im Juni 2020 beschlossene Konjunkturprogramm der Bundesregierung in ihrer Gesamtheit als notwendig, zweckmäßig und dem Umfang nach angemessen, um die deutsche Konjunktur zu stabilisieren. Wirtschaftspolitische Entscheidungen in Krisen-Situationen stehen vor einem Konflikt zwischen Zielgenauigkeit und Reaktionsschnelligkeit. Bei einigem Für und Wider im Einzelnen sind die beschlossenen Maßnahmen geeignet, die Investitionstätigkeit und den Konsum zu stützen und damit die Erholung zu beschleunigen. Die Mehrwertsteuersenkung und der Kinderbonus begünstigen zudem Haushalte mit mittleren und geringen Einkommen, die besonders von der Krise betroffen sind.

Title: Recovery Loses Momentum - Economy and Politics Still Shaped by the Pandemic

Abstract: The corona pandemic has left substantial marks on the German economy and its impact is more persistent than presumed in the spring. In their autumn reports, leading German economic research institutes have revised their economic outlook downwards by roughly one percentage point for both this and next year. They now expect gross domestic product to fall by $5.4 \%$ in 2020 (previously $4.2 \%$ ) and to grow by $4.7 \%$ (5.8\%) in 2021 and $2.7 \%$ in 2022. The downgrade of the forecast follows a more pessimistic assessment of the recovery, which is being held back by those sectors that are particularly dependent on social contacts. The precrisis level of output will not be reached until the end of 2021 with GDP remaining at $2.5 \%$ below the level that would have prevailed without the pandemic. Despite massively falling back on shorttime working schemes, an estimated 820,000 jobs were lost due to the crisis. The government will run a record high budget deficit of 183 billion euros in 2020. In 2021 and 2022, deficits will remain substantial at 118 billion euros and 92 billion euros, respectively.

JEL Classification: E32, E66 